1 Genomic surveillance of antimicrobial resistance shows cattle are a moderate source of multi-drug resistant non-typhoidal Salmonella in Mexico

6 Enrique Jesús Delgado-Suárez ${ }^{1}$, Tania Palós-Guitérrez¹ ${ }^{1}$ Francisco Alejandro Ruíz-

7 López¹, Cindy Fabiola Hernández Pérez², Nayarit Emérita Ballesteros-Nova1, Orbelín

8 Soberanis-Ramos ${ }^{1}$, Rubén Danilo Méndez-Medina ${ }^{1}$, Marc W. Allard ${ }^{3}$, María Salud

$$
\text { Rubio-Lozano }{ }^{1^{*}}
$$

${ }^{1}$ Facultad de Medicina Veterinaria y Zootecnia, Universidad Nacional Autónoma de

${ }^{2}$ Centro Nacional de Referencia de Plaguicidas y Contaminantes, Dirección General de Inocuidad Agroalimentaria, Acuícola y Pesquera, Servicio Nacional de Sanidad, Inocuidad y Calidad Agroalimentaria, Estado de México, México

18 E-mail: msalud65@gmail.com (MSRL)

\title{
20 Abstract
}

21 Multi-drug resistant (MDR) non-typhoidal Salmonella (NTS) is a major public health

22 concern globally. This study reports antibiotic susceptibility testing and genotypic

23 antimicrobial resistance (AMR) profiles of NTS isolates from bovine lymph nodes $(n=48)$ 
24 and ground beef $(n=29)$. Furthermore, we compared genotypic AMR data of our isolates

25 with those of publicly available NTS genomes from Mexico $(n=1637)$. The probability of

26 finding MDR isolates was higher in ground beef as compared to lymph nodes: $x^{2}=12.0$,

$27 \mathrm{P}=0.0005$. The most common resistant phenotypes involved tetracycline $(40.3 \%)$,

28 carbenicillin (26.0\%), amoxicillin-clavulanic acid (20.8\%), chloramphenicol (19.5\%) and

29 trimethoprim-sulfamethoxazole (16.9\%), while over $55 \%$ of the isolates showed

30 decreased susceptibility to ciprofloxacin and $26 \%$ were MDR. Occurrence of MDR

31 isolates was strongly associated with NTS serovar $\left(x^{2}=24.5, P<0.0001\right)$, with

32 Typhimurium accounting for $40 \%$ of MDR strains. Most of these (9/10), carried

33 Salmonella genomic island 1, which harbors multiple AMR genes (aadA2, blaCARB-2,

34 floR, sul1, tetG) that confer a penta-resistant phenotype. Moreover, $100 \%$ of MDR

35 isolates had mutations in the ramR gene. Among public NTS isolates from foods and

36 clinical cases in Mexico to date, those from cattle had the highest proportion of MDR

37 genotypes. Our results suggest attaining significant improvements in AMR meat safety

38 may require the identification and removal (or treatment) of product harboring MDR

39 NTS, instead of screening for Salmonella spp. or for isolates showing resistance to

40 individual antibiotics. In that sense, massive integration of whole genome sequencing

41 (WGS) technologies in AMR surveillance provides the shortest path to accomplish these 42 goals.

\section{Introduction}

45 For decades, experimental data has supported concerns that antimicrobials use (AMU)

46 in animal production is a key factor contributing to the ever-growing crisis of bacterial 
47 antimicrobial resistance (AMR). However, there is limited evidence linking food

48 consumption with AMR emergence in humans [1, 2]. Moreover, an increasing number of

49 studies have found that AMU in food animals has a limited impact on the AMR profile of

50 foodborne pathogens [3-6]. While this may be true in developed countries, the situation

51 is likely different in low and middle-income countries (LMIC) where controls of AMU in

52 veterinary practice and human health are less rigorous. Therefore, in the context of an

53 intense trade of foods between countries differing in AMR food safety, it is vital to

54 identify relevant sources of AMR pathogens along the food chain. This would help to

55 prevent their dissemination, as well as human exposure to multi-drug resistant (MDR)

56 bacteria, which is a global public health issue.

57 In Mexico, source attribution of foodborne illnesses is in an early stage. Nonetheless,

58 official epidemiological surveillance reports high incidence rates for infections commonly

59 transmitted through foods. That of non-typhoidal salmonellosis has been above 60

60 cases per 100 thousand inhabitants in the last 5 years [7]. In addition, recent studies

61 conducted in Mexico show non-typhoidal Salmonella (NTS) contamination is unusually

62 high (15 to nearly 100\% positive samples) in bovine lymph nodes, beef carcasses and

63 ground beef, with isolates exhibiting rates of resistance that vary from 30 to nearly $98 \%$,

64 across several important antimicrobial classes (i. e. penicillins, aminoglycosides,

65 cephalosporins, quinolones) $[8,9]$. This evidence suggests beef is likely to play a role

66 as a relevant reservoir of foodborne MDR salmonellosis in Mexico. Especially, if

67 considering it is often involved in salmonellosis outbreaks in countries with lower NTS

68 contamination rates $[10,11]$.

69 The increasing availability of whole genome sequencing (WGS) technologies have

70 helped to improve genomic surveillance as it provides a high-resolution method for the 
71 characterization of an organism features. Regarding AMR in NTS, however, most

72 research from LMIC deal with phenotypes [12-16]. Although these studies raise

73 concerns, they do not provide insights into the genetic basis of AMR, its evolution or

74 dissemination within bacterial populations. Such information is crucial to devise new

75 strategies to contain the dissemination of AMR pathogens. However, it can best be

76 obtained by addressing phenotypic and genomic profiling of AMR simultaneously, an

77 area with a limited number of studies in Mexico and other countries [17].

78 In the present investigation, we conducted antibiotic susceptibility testing and WGS of

7977 NTS isolates collected in the course of a previous research project involving bovine

80 lymph nodes $(n=800)$ and ground beef $(n=745)$ across a two-year sampling period [18].

81 Assembled genomes were used to predict the AMR genomic profiling of NTS isolates,

82 and this was further compared to their corresponding AMR phenotypes. We also

83 conducted comparative genomics of AMR genotypes of publicly available NTS isolates

84 collected in Mexico from bovines, vegetables, seafood, aquatic environments, human

85 stools and clinical cases. Consequently, we managed to thoroughly characterize the

86 role of cattle as a reservoir of NTS harboring AMR genes of human clinical significance.

87 Moreover, we identified the dominant genetic determinants that sustain resistance

88 against specific antibiotic classes, as well as MDR phenotypes.

90 Materials and Methods

91 Animal Care and Use Committee approval was not obtained for this study since live 92 animals were not directly involved in the experiment. 


\section{Salmonella isolates}

94 We used 77 strains from a previous study conducted by our research group [18]. These

95 were isolated from bovine lymph nodes $(L N, n=800)$ and ground beef $(G B, n=745)$

96 collected between April 2017 and December 2018. Each isolate was obtained from a

97 different sample across the two-year sampling period.

99 Antibiotic Susceptibility Testing (AST)

100 The phenotypic AMR profile of NTS isolates was determined with a panel of 13

101 antibiotics included in the WHO list of critically important and highly important

102 antimicrobials [19]. We used the disk diffusion method [20], with the Bencton Dickinson

103 AST test disks and concentrations reported in Table 1.

105 Table 1. Antimicrobial agents tested, their concentrations and zone diameter clinical

106 break points used in the AST

Zone diameter breakpoint ${ }^{1}, \mathrm{~mm}$

\begin{tabular}{lccc} 
Antimicrobials & $\mathrm{C}, \mu \mathrm{g}^{2}$ & $\mathrm{R}$ & $\mathrm{I}$ \\
\cline { 3 - 4 } & & $\leq 14$ & $15-16$ \\
Amikacin (AMK) & 30 & $\leq 12$ & $13-14$ \\
Gentamicin (GEN) & 10 & $\leq 19$ & $20-22$ \\
Amoxicillin/clavulanic acid (AMC) & 100 & $\leq 13$ & $14-17$ \\
Cefotaxime (CTX) & $20 / 10$ & $\leq 22$ & $23-25$ \\
Ceftriaxone (CRO) & 30 & $\leq 19$ & $20-22$ \\
Cefepime (FEP) & 30 & $\leq 18$ & $19-24$
\end{tabular}




$\begin{array}{lccc}\text { Imipenem (IPM) } & 10 & \leq 19 & 20-22 \\ \text { Ertapenem (ETP) } & 10 & \leq 18 & 19-21 \\ \text { Meropenem (MEM) } & 15 & \leq 19 & 20-22 \\ \text { Chloramphenicol (CHL) } & 30 & \leq 12 & 13-17 \\ \text { Trimethoprim-sulfamethoxazole (SXT) } & 1.25 / 23.75 & \leq 10 & 11-15 \\ \text { Ciprofloxacin (CIP) } & 5 & \leq 20 & 21-30 \\ \text { Tetracycline (TET) } & 30 & \leq 11 & 12-14\end{array}$

$107{ }^{1}$ Criteria to consider isolates as clinically resistant $(R)$ or intermediate (I) [21]. For

108 meropenem, we also considered epidemiological cutoff values (ECOFF) set by

109 EUCAST to classify strains as wild-type $(>27 \mathrm{~mm})$ or non-wild-type $(\leq 27 \mathrm{~mm})$ [22].

$110 \quad{ }^{2}$ Antimicrobial's disk concentration.

112 Isolates were classified as susceptible, intermediate or resistant, according to the

113 Clinical Laboratory Standards Institute (CLSI) guidelines [21]. Strains of Escherichia coli

114 ATCC 8739, Enterococcus fecalis ATCC 29212, Staphylococcus aureus ATCC 25923,

115 and Pseudomonas aeruginosa ATCC 9027 were used as quality control organisms.

116 Isolates showing intermediate and resistance phenotypes were considered as non-

117 susceptible in this study. Likewise, isolates showing resistance to $\geq 3$ antimicrobial

118 classes were classified as MDR [23]. The detailed AST protocol is available at

119 protocols.io (doi: dx.doi.org/10.17504/protocols.io.bpypmpvn).

121 Whole-genome sequencing and genome assembly

122 Genomic DNA (gDNA) was extracted from fresh colonies grown overnight at $37^{\circ} \mathrm{C}$ in 123 tryptic soy broth. For that purpose, we used the Roche PCR High Purity Template 
124 Preparation Kit (Roche México, Mexico City, Mexico), following manufacturer's

125 instructions. Subsequently, gDNA was quantified using a Qubit 3.0 Fluorometer

126 (Thermo Fisher Scientific México, Mexico City, Mexico). Afterwards, sequencing

127 libraries were prepared from 1 ng gDNA using the Nextera XT Library Preparation Kit

128 v.3 (Illumina) and sequenced on the Illumina NextSeq system (paired end 2 x $150 \mathrm{bp}$

129 insert size). Raw sequences are publicly available at the National Center for

130 Biotechnology Information (NCBI). The accession numbers and metadata are listed in

131 supplementary S1 Table.

132 The quality of raw reads was first assessed with FastQC [24] and we used Trimmomatic

133 [25] to filter poor-quality reads and Illumina adaptors. Trimmed sequences were

134 analyzed again with FastQC to ensure only high-quality reads (i. e. Q $\geq 30$ ) were used for

135 de novo genome assembly. Finally, genome assembly was conducted in the PATRIC

136 web server [26] using the SPAdes algorithm [27].

138 Genotypic AMR profiling and comparative genomics

139 AMR genes and point mutations associated with AMR were predicted with the aid of

140 AMRFinderPlus program version 3.8 using assembled genomes [28]. The study also

141 aimed to compare the genetic AMR profile of our isolates in the context of NTS

142 population circulating in the country. For that purpose, we identified all Salmonella

143 isolates from Mexico that were publicly available at NCBI as of September 21, 2020

$144(n=2400)$. Among these, we selected groups of isolates of known isolation source:

145 bovines ( $n=114$, including the 77 from this study), vegetables $(n=1,064)$, seafood

$146(n=129)$, aquatic environment $(n=385)$, and human stools/clinical cases $(n=22)$. The

147 human isolates group will be referred to as "human clinical cases" from now on. The full 
148 list of accessions and metadata of these isolates is provided in supplementary S2

149 Table. AMR genotypes of these isolates were collected from the NCBI Pathogen

150 Detection web site (https://www.ncbi.nlm.nih.gov/pathogens), which are generated by

151 the AMRFinderPlus database and program.

152 Most serovar Typhimirum isolates (8/10) exhibited a penta-resistant phenotype similar

153 to that reported for the Typhimurium DT104 strain, which is sustained by Salmonella

154 genomic island 1 (SGI1) [29]. Hence, we conducted a BLAST atlas analysis to

155 determine if the MDR profile of these isolates was associated with this genomic feature.

156 For that purpose, we used assembled genomes at the GView web server [30],

157 configured as follows: expect e-value cutoff $=0.001$, genetic code=bacterial and plant

158 plastid, alignment length cutoff $=50$, percent identity cutoff $=70$ and tblastx as the BLAST

159 program. The SGI1 reference sequence (AF261825.2) was collected from the

160 Pathogenicity Island Database [31]. Furthermore, considering the epidemiological

161 importance of this serovar, we also analyzed the whole set of Typhimurium isolates

162 from Mexico deposited at NCBI ( $n=38$, refer to S3 Table for accession numbers and

163 AMR genotypes of this group of isolates). This analysis was performed in order to

164 estimate how common MDR profiles are in strains of this serovar circulating in Mexico.

166 Plasmid profiling

167 Plasmids are known as strong contributors to AMR dissemination. Hence, we used

168 PlasmidFinder 2.1 [32] to predict the isolates plasmid profile using assembled genomes

169 and a threshold identity of $95 \%$. When plasmid replicons were detected, we collected

170 each plasmid's reference sequence from NCBI and mapped it against assembled

171 genomes. If most plasmid sequences (>70\%) were represented in a genome and the 
172 genomic context of genes matched that of the plasmid, these isolates were proposed to

173 carry the predicted plasmid.

175 Data analysis

176 We used Chi-square tests and odds ratio calculations to determine if there was an

177 association between AMR profiles (both phenotypic and genotypic) and NTS serovars

178 and/or isolation sources. These analyses were performed both with our experimental

179 isolates, as well as with the additional public NTS genomes from Mexico included in the

180 study.

181 We also conducted a correlation analysis between phenotypic and genotypic AMR

182 results. Moreover, the genomic AMR profile of public NTS from Mexico was analyzed

183 with the aid of a heatmap. For that purpose, we first determine the number of isolates

184 from each source harboring specific AMR genes. Using these figures, we calculated the

185 proportion of isolates from each source having the feature and used these data to

186 generate a heatmap with the Heatmapper software [33].

\section{Results}

189 Phenotypic and genotypic antimicrobial resistance profiles

190 Approximately three quarters of the isolates were non-susceptible to at least one

191 antibiotic class, while $26 \%$ were susceptible to all the tested antibiotics (Fig 1). The

192 most common resistant phenotypes included tetracycline (40.3\%), carbenicillin (26.0\%),

193 amoxicillin-clavulanic acid (20.8\%), chloramphenicol (19.5\%) and trimethoprim-

194 sulfamethoxazole (16.9\%). Resistance to cephalosporins and aminoglycosides was less 
195 frequent (1.3-7.8\% across these antibiotic classes), while for carbapenems only

196 intermediate resistance was observed at a frequency of 1.3 and $9.1 \%$ for ertapenem

197 and imipenem, respectively. On the other hand, although only one isolate resisted

198 ciprofloxacin, $54.5 \%$ of the strains showed intermediate resistance to this drug.

199 Fig 1. AMR profile of 77 Salmonella isolates from lymph nodes (LN) and ground beef

200 (GB). Cells are color coded according to antibiotic class: orange, aminoglycosides; blue, 201 beta-lactams; purple, phenicols; green, sulfonamides; red, fluoroquinolones; black, 202 tetracyclines. Resistant strains are indicated with "R" and intermediate with "I". For 203 genotypes, cells with the antibiotic class color indicate presence of the gene. Genes in 204 gray are not associated with any antibiotic class included in the AST panel. Antibiotic 205 abbreviations are as follows: amikacin (AMK), gentamycin (GEN), carbenicillin (CB), 206 amoxicillin-clavulanic acid (AMC), cefotaxime (CTX), ceftriaxone (CRO), cefepime (FEP), 207 meropenem (MEM), ertapenem (ETP), imipenem (IMP), chloramphenicol (CHL), 208 trimethoprim-sulfamethoxazole (SXT), ciprofloxacin (CIP), tetracycline (TET).

210 The rate of MDR strains was $26 \%$, with the most common MDR profiles involving

211 penicillins, chloramphenicol, trimethoprim-sulfamethoxazole, ciprofloxacin and

212 tetracycline. Strikingly, there was one serovar Typhimurium isolate that resisted all

213 antibiotic classes but carbapenems. In fact, the probability of isolates having MDR

214 profiles was significantly higher in strains of serovar Typhimurium as compared to other

215 serovars $(\mathrm{OR}=45.8,95 \mathrm{Cl} 5.3-399.2, \mathrm{P}<0.0001)$. Likewise, there was a higher probability

216 of finding MDR strains in ground beef as compared to lymph nodes (OR=6.5 $95 \mathrm{Cl} 2.1$ -

217 20.1, $P=0.0005)$. 
218 The WGS-based in-silico prediction of non-susceptible phenotypes was generally good,

219 as shown by the strong correlation between AMR genotypes and phenotypes (Fig 2).

221 Fig 2. Overall correlation of phenotypic resistance with the presence of antimicrobial

222 resistance genes for the 77 Salmonella isolates $(r=0.979, P<0.0001)$. Antibiotic

223 abbreviations: ciprofloxacin (CIP), tetracycline (TET), carbenicillin (CB), amoxicillin-

224 clavulanic acid (AMC), chloramphenicol (CHL), trimethoprim-sulfamethoxazole (SXT),

225 imipenem (IMP), ceftriaxone (CRO), cefotaxime (CTX), gentamycin (GEN), amikacin

226 (AMK), cefepime (FEP), meropenem (MEM), ertapenem (ETP).

228 Genomic AMR profiling also identified the presence of several additional resistance

229 genes that are not associated with any specific antimicrobial included in the AST panel.

230 Particularly, those encoding resistance to fosfomycin (fos $A 7.7$ ), quaternary ammonium

231 compounds (qacEDelta1), as well as components of the multidrug and metal RND-type

232 efflux complex ( $m d s A B)$.

233 Assembled genomes were also analyzed for point mutations associated with resistance.

234 However, the occurrence of mutations was mostly inconsistent with the observed

235 phenotypes (Table 2).

237 Table 2. Relative frequency of chromosomal point mutations detected in the studied

238 Salmonella isolates $(n=77)$ and their associated resistance phenotypes ${ }^{1}$

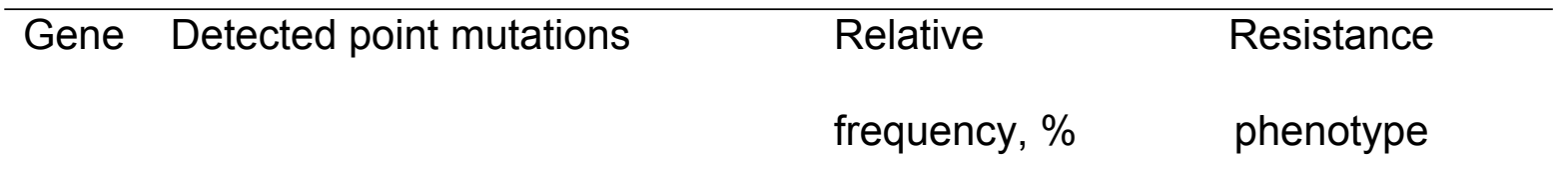




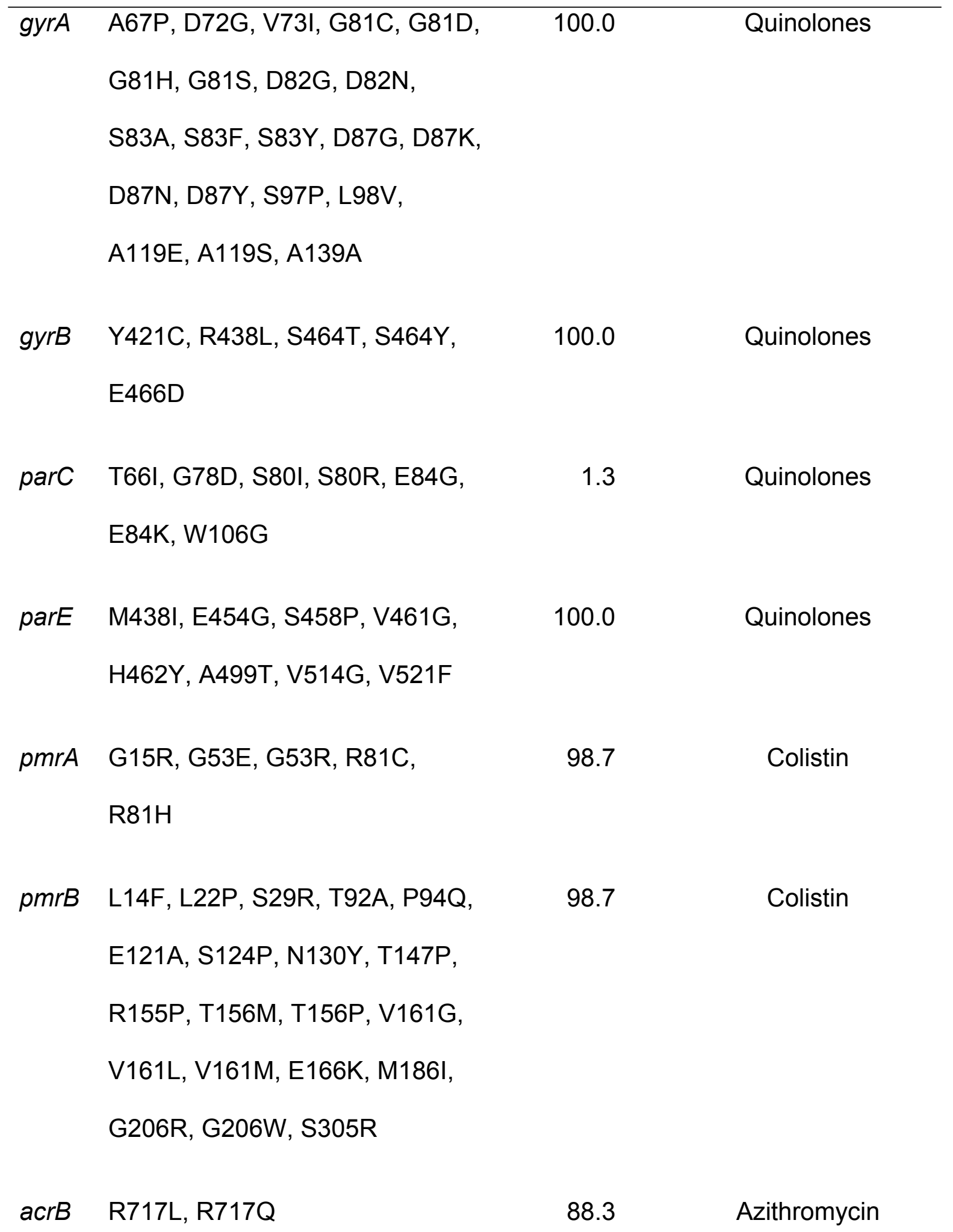


soxR R20H, G121D

soxS E52K

ramR T18P, G25A, R46P, T50P, Y59H,

M84I, E160D
100.0 Penicillins, phenicols,

quinolones, rifampin,

tetracyclines

100.0 Penicillins, phenicols,

quinolones, rifampin,

tetracyclines

68.8 Penicillins, phenicols,

quinolones, rifampin,

tetracyclines

241 For instance, all isolates carried multiple mutations in the quinolone resistance-

242 determining region (QRDR), gyrAB and parE genes, regardless of being susceptible or

243 not to ciprofloxacin. Likewise, $100 \%$ isolates had mutations in soxRS genes, which are

244 known to confer MDR profiles [34]. Still, only $26 \%$ of isolates were classified as MDR.

245 Conversely, mutations in ramR were strongly associated with the occurrence of MDR

246 strains $\left(X^{2}=17.7, P<0.0001\right)$.

247 Genomic analysis also revealed additional widespread mutations. Those of $p m r A B$

248 genes, which are associated with colistin resistance, were present in all isolates.

249 Likewise, mutations in the acrB gene, which confer resistance to azithromycin, were

250 detected in $88.3 \%$ of the strains. These two drugs, however, were not included in the

251 AST panel. Below, a detailed description of the relationship between AMR phenotypes 
252 and genotypes will be presented for each of the tested antibiotic classes, as well as for

253 MDR strains.

\section{Aminoglycoside resistance}

256 Aminoglycoside resistance was supported by the presence of genes encoding

257 enzymatic inactivation mechanisms, such as phosphorylation (several aph alleles) and

258 adenylation (aadA2 gene). Comparative analysis with public Salmonella genomes

259 revealed a strong association between aminoglycoside resistant and the source of the

260 isolates $\left(\mathrm{X}^{2}=89.2, \mathrm{P}<0.0001\right)$

261 Clinical strains had the highest proportion of aminoglycoside resistance genotypes (Fig

2623 ) and were more likely to carry AMR genes against this antibiotic class as compared to

263 those from food related sources ( $\mathrm{OR}=7.7,95 \mathrm{Cl} 3.2-18.3)$. Among non-clinical samples,

264 bovines were the most significant contributors of aminoglycoside resistant NTS

$265(\mathrm{OR}=5.0,95 \mathrm{Cl} 3.1-7.9)$. In general, however, the resistance profile was similar across

266 sources, with a predominance of aadA and aph genes over the acc alleles.

268 Fig 3. Comparative genomic AMR profile of public NTS isolated from human clinical

269 cases, foods, and food related sources in Mexico. Refer to S2 Table for accessions and

270 metadata of isolates included in this analysis.

272 Beta-lactam resistance

273 Experimental isolates harbored only the Ambler's class A beta-lactamase-encoding

274 genes blaCARB-2 and blaTEM-1. These enzymes are known to confer resistance to all

275 penicillins, as well as first, second and third generation cephalosporins [35]. The 
276 blaCARB-2 gene was the most abundant among isolates showing resistance to

277 penicillins, especially in those of serovar Typhimurium (9/10). One isolate of serovar

278 Anatum and one of monophasic (14,[5],12:i:-) Typhimurium also carried blaTEM-1,

279 which encode another extended-spectrum beta-lactamase (ESBL) that hydrolyzes

280 penicillins and first-generation cephalosporins [35]. There were 11 non-susceptible

281 isolates that did not carry any of the known ESBL-encoding genes. Strikingly, however,

28210 out of these isolates had ramR mutations, which are associated with resistance

283 towards several antibiotic classes, including penicillins.

284 Despite eight isolates were non-susceptible to third generation cephalosporins (3GC)

285 and one to both $3 \mathrm{GC}$ and fourth generation cephalosporins (4GC), none carried ESBL-

286 encoding genes associated with these phenotypes. As observed with penicillins,

287 mutations in ramR appeared to be associated with non-susceptibility to cephalosporins

288 since $100 \%$ of non-susceptible isolates to $3 \mathrm{GC} / 4 \mathrm{GC}$ had these mutations.

289 Non-susceptibility to carbapenems was the least frequent among the tested antibiotic

290 classes. No isolate resisted meropenem, while only one showed intermediate resistance

291 to ertapenem and seven to imipenem. Again, no carbapenemase-encoding genes were

292 found in the genome of any of the non-susceptible isolates. Interestingly, however,

293 when using the epidemiological cutoff value of the inhibition zone ( $\leq 27 \mathrm{~mm}$ ) set for

294 meropenem in the European Union [22], 17 isolates (22\%) were classified as non-wild

295 type, which indicates some mechanism of carbapenem resistance could be emerging in

296 the studied population.

297 In relation to other public Salmonella genomes from Mexico, clinical cases were also the

298 main source of beta-lactam-resistant Salmonella: $\mathrm{X}^{2}=72.5, \mathrm{P}<0.0001, \mathrm{OR}=12.8,95 \mathrm{Cl}$ 
4.5-36.5 (Fig 3). Again, bovines were the most important reservoir, among food related

300 samples, of these AMR genotypes: $x^{2}=39.3, P<0.0001, \mathrm{OR}=6.1,95 \mathrm{Cl} 3.0-12.7$.

301 Class-A extended spectrum beta-lactamase (ESBL)-encoding genes (i. e. blaCARB,

302 blaTEM) were the most abundant among isolates from all sources. Genes encoding

303 class-C ESBLs (i. e. blaCMY) were also present in a small proportion of isolates from

304 clinical cases (4.6\%), vegetables (0.3\%), and aquatic environments (1.6\%), while the

305 blaOXA gene, which encodes a class-D ESBL, was only detected in $9 \%$ of clinical

306 strains. Fortunately, class-B ESBL-encoding genes were not detected in the genome of

307 the studied public isolates.

\section{Fluoroquinolone resistance}

310 The main resistance mechanism we detected was that encoded by plasmid-mediated

311 quinolone resistance (PMQR) genes, such as qnrB19 and qnrA1, involved in quinolone

312 target protection (DNA gyrase). This genomic feature, which is known to confer low-

313 level quinolone resistance, was strongly associated with the intermediate resistance

314 against ciprofloxacin observed in our study $\left(\mathrm{X}^{2}=36.8, \mathrm{P}<0.0001\right)$. As mentioned before,

315 we did not find statistical association between point-mutations in the QRDR and the

316 observed phenotypes.

317 Among public Salmonella genomes, those from cattle origin were the major source of

318 PMQR genes (Fig 3), with $32.5 \%$ of these isolates carrying $q n r$ alleles $\left(x^{2}=196.9\right.$,

$319 \mathrm{P}<0.0001, \mathrm{OR}=13.8,95 \mathrm{Cl}$ 8.5-22.2). Conversely, the proportion of PMQR-positive

320 isolates was $18.2 \%$ in clinical strains, while it ranged from 1.6 to $6.0 \%$ across isolates

321 from vegetables, seafood, and aquatic environments. Public genomes encoded

322 additional quinolone resistance factors, such as efflux mechanisms (oqxAB genes). 
323 However, these genes were not as abundant as qnr alleles and were restricted mostly

324 to clinical strains (13.6\%) and a very small proportion of vegetable isolates $(0.4 \%)$.

\section{Chloramphenicol resistance}

327 Resistance to chloramphenicol in experimental isolates was mainly associated with

328 efflux mechanisms (floR gene). This gene was present in most isolates of serovar

329 Typhimurium (8/10), accounting for over $50 \%$ of chloramphenicol non-susceptible

330 phenotypes. We detected a second resistance mechanism (antibiotic inactivation),

331 encoded by a chloramphenicol acetyltransferase gene (catA2). However, this gene was

332 present just in one isolate of serovar Give. Moreover, there were six non-susceptible

333 isolates that were predicted as genotypically susceptible. Again, ramR mutations were

334 strongly associated with chloramphenicol resistance $\left(x^{2}=8.1, P=0.0045\right), O R=21.195 \mathrm{Cl}$

$335 \quad 1.2-368.4$

336 As observed for previous antibiotic classes, there was a strong association between

337 source and the proportion of phenicol-resistant Salmonella genotypes $\left(x^{2}=187.7\right.$,

$338 \mathrm{P}<0.0001$ ) among public NTS isolates. Clinical strains were more likely to carry

339 phenicols resistance genes than isolates from any other source: $\mathrm{OR}=11.7,95 \mathrm{Cl}$ 4.6-

340 29.7. Likewise, cattle were the dominant reservoir, among food related samples, of

341 these AMR genotypes (OR=15.6, 95Cl 8.9-27.1). Still, the genomic AMR profile was

342 similar across sources (Fig 3), with a predominance of efflux factors (i. e. floR) over

343 enzymatic inactivation mechanisms (i. e. cat $A B$ ). 


\section{Folate pathway inhibitors resistance}

345 Regarding folate pathway inhibitors, the most abundant resistance mechanism among 346 experimental isolates was that encoded by sul alleles (i. e. sul1 and sul2), which were 347 present in 11 isolates. Conversely, just one isolate carried the dfrA12 gene along with 348 the sul1 gene. Overall, there was a discrete proportion of experimental isolates that 349 resisted trimethoprim-sulfamethoxazole (16.9\%).

350 The resistance profile of public genomes again revealed isolates of bovine origin are 351 more likely to carry trimethoprim-sulfonamide resistance genes as compared to those

352 from vegetables, seafood and aquatic environments $\left(X^{2}=142.0, P<0.0001\right), O R=11.7$

$35395 \mathrm{Cl} 7.1-19.2$ (Fig 3). In all these sources, sul and dfrA alleles were the most common.

354 In general, however, clinical isolates represent a more significant source of

355 trimethoprim-sulfonamide resistant Salmonella as compared to those from food related

356 isolates $\left(x^{2}=175.9, P<0.0001\right), O R=11.595 C l 4.7-28.2$.

\section{Tetracycline resistance}

359 Among experimental isolates, resistance to tetracycline was mainly associated with the

360 presence of genes encoding efflux mechanisms (i. e. tetABCG). There were seven non-

361 susceptible isolates that did not carry any of the known tetracycline resistance genes.

362 However, all these isolates carried ramR mutations, which are associated with MDR

363 profiles involving tetracyclines and other antibiotic classes [36].

364 Comparison with public Salmonella genomes revealed the highest proportion of

365 tetracycline-resistance genotypes corresponded to bovine sources $\left(x^{2}=189.1\right.$,

$366 \mathrm{P}<0.0001), \mathrm{OR}=10.995 \mathrm{Cl} 7.1-16.9$, as compared to vegetables, seafood, aquatic

367 environments and even clinical cases (Fig 3). Regarding the genomic AMR profile, 
368 isolates from all sources carried some variants of the same efflux-mediated resistance

369 determinants (tet alleles).

\section{MDR profiles}

372 Among experimental isolates, MDR profiles were associated with the presence of a

373 resistance island and, to a lesser extent, the occurrence of mutations in their genomes.

374 For instance, most serovar Typhimurium isolates (9/10) carried SGI1. This genomic

375 island harbors a class-1 integron containing multiple gene cassettes (i. e. aadA2,

376 blaCARB-2, floR, sul1, tetG), which explains the observed MDR phenotypes of these

377 isolates (Fig 4). This feature appears to be common in S. enterica ser. Typhimurium

378 circulating in Mexico. The analysis of the whole set of Typhimurium isolates from

379 Mexico deposited at NCBI $(n=38)$ showed $55.3 \%$ of them have MDR genotypes. Among

380 these, nearly $70 \%$ harbor multiple AMR genes associated with the ACSSuT phenotype.

381 Refer to supplementary S3 Table for accession numbers and AMR genotypes of this

382 group of isolates.

383

384 Fig 4. BLAST atlas analysis of SGI1 of 10 serovar Typhimurium isolates and one

385 monophasic Typhimurium. The black slot corresponds to the backbone and the brown

386 inner ring to the reference sequence. The most inner ring shows gene annotation, with

387 AMR genes highlighted in bold text. The outer rings correspond to the serovars and

388 sample names indicated. Regions with shared synteny between genomes and

389 reference sequence are filled with intense color while blank spaces indicate lack of

390 synteny. Refer to S1 Table for accession numbers and metadata of isolates. 
392 As mentioned before, mutations in the ramR gene (Table 2) were strongly associated

393 with MDR phenotypes as well. In fact, the probability of finding MDR strains was 50.5

394 times higher (95Cl 2.9-881.3) in isolates carrying ramR mutations as compared to those

395 lacking them.

396 Interestingly, plasmids had a rather discrete contribution to AMR phenotypes in general

397 (Table 3). Only one third of the studied isolates $(n=26)$ were predicted to carry seven

398 different plasmids. Of these, three were small plasmids harboring few replication-related

399 genes. The most abundant plasmid was the Salmonella virulence plasmid (pSLT),

400 which was detected in all serovar Typhimurium isolates. The pSLT is a hybrid plasmid

401 that carries a class-1 integron with several AMR gene cassettes against beta-lactams

402 (blaTEM), chloramphenicol (catA), aminoglycosides (aadA1, strAB), and folate pathway

403 inhibitors (sul1, sul2, dfrA1). However, the integron carried by our serovar Typhimurium

404 isolates had a genomic context matching that of SGI1 instead of pSLT (see Fig 4 and

405 supplementary S1 Figure). 
406 Table 3. General features of the plasmids predicted per Salmonella serovar

\begin{tabular}{|c|c|c|c|c|}
\hline $\begin{array}{l}\text { Plasmid (NCBI } \\
\text { accession) }\end{array}$ & Size, bp & $\begin{array}{l}\text { Incompatibility } \\
\text { group }\end{array}$ & Plasmid AMR genes $^{1}$ & Serovar $(n)^{2}$ \\
\hline$\overline{\mathrm{pSLT}}$ (FN432031) & 117,047 & $\operatorname{lncFIB}(S)$ & $\begin{array}{c}\text { aadA1, blaTEM, catA1, dfrA1, strAB, sul1, } \\
\text { sul2 }\end{array}$ & Typhimurium (10) \\
\hline pK245 (DQ449578) & 98,264 & IncR & $\begin{array}{c}\text { aacC2, blaSHV2A, catA2, dfrA14, strAB, } \\
\text { sul2, tetD, tetR }\end{array}$ & Give (1) \\
\hline R64 (AP005147) & 120,826 & Incl1 & tet $A C D$, strAB & $\begin{array}{l}\text { Fresno (4) } \\
\text { Anatum (1) }\end{array}$ \\
\hline pOLA52 & 51,602 & $\operatorname{IncX} 1$ & blaTEM, oqxAB & 1,4,[5],12:i:- (1) \\
\hline RSF1010 & 8,684 & IncQ1 & -- & 1,4,[5],12:i:- (1) \\
\hline pBS512_2 & 2,089 & -- & -- & $\begin{array}{l}\text { Anatum (2) } \\
\text { London (4) } \\
\text { Reading (1) }\end{array}$ \\
\hline pVCM01 & 1981 & --- & -- & Anatum (2) \\
\hline
\end{tabular}

$407{ }^{1}$ Only AMR genes in bold were present in both the plasmid and the genomes.

$408{ }^{2}$ Number of isolates of the same serovar carrying replicons of the plasmid. 
409 Among the plasmids detected, the pK245 has the strongest resistance profile, carrying

410 a class-1 integron with a sole resistance cassette (dfrA14), as well as another seven

411 AMR genes (tetAR, sul2, strAB, catA2, blaSHV-2) distributed across the plasmid

412 sequence. However, it is unlikely that pK245 plasmid was actually present in that

413 isolate. Only $30 \%$ of this plasmid aligned to the genome of the single isolate (a serovar

414 Give strain) carrying its replicons. Moreover, this isolate carried a chromosomal class-1

415 integron with seven resistance cassettes in a genomic context different from that of

416 pK245 (see supplementary S2 Figure).

417 Replicons of the resistance plasmid R64 were also found in the genomes of serovar

418 Fresno isolates $(n=4)$, as well as one serovar Anatum isolate. This plasmid carries AMR

419 genes against tetracyclines (tetADR) and aminoglycosides (strAB). Although over $70 \%$

420 of R64 aligned to the assembled genomes, none of its AMR genes were found in the

421 genomes of isolates carrying its replicons, except tet $A$, which was detected in the

422 serovar Anatum isolate (see supplementary S3 Figure). Finally, the monophasic

423 Typhimurium isolate carried replicons of the pOLA52 plasmid. This is also a hybrid

424 plasmid that harbors genes encoding a type IV secretion system, as well as AMR genes

425 against quinolones (oqxAB) and beta-lactams (blaTEM). Although over $70 \%$ of this

426 plasmid aligned to the referred genome, only the blaTEM gene was also present in the

427 isolate, while oqxAB genes were missing (see supplementary S4 Figure).

428 Finally, the analysis of public genomes showed bovines and clinical cases are the most

429 important sources, among those studied, of MDR NTS genotypes (Fig 3). In the other

430 matrices, the proportion of MDR genotypes ranged from 2 to nearly $6 \%$. 


\section{Discussion}

432 Our results confirmed previous observations of widespread resistance to older

433 antibiotics (i. e. tetracyclines, penicillins) among NTS from different sources. For

434 instance, in Mexico, previous studies from over 10 years ago, as well as recent

435 research, report these phenotypes at very high frequencies (up to $90 \%$ or higher) in

436 beef isolates $[12,15,17,37-39]$. Likewise, these AMR phenotypes are also common in

437 NTS isolated in developed countries, a phenomenon that is thought to be driven by the

438 use of these antimicrobials in food-producing animals [40].

439 Regarding tetracyclines, selective pressure has likely resulted in the extensive

440 acquisition of efflux mechanisms (tetABCG alleles), which are usually carried either in

441 plasmids or in the chromosome of NTS [41]. Our results also support certain point

442 mutations could sustain this phenotype in isolates lacking tet alleles, providing evidence

443 of convergent evolution. As observed here, $100 \%$ of isolates that did not carry tet alleles

444 had ramR mutations. These mutations are known to induce over expression of the

445 AcrAB-RND efflux system [42], which confer resistance against multiple drugs, including

446 tetracyclines.

447 Strikingly, results showed cattle seemed more relevant than any other source, including

448 clinical cases, as a source of tetracycline resistance NTS. Although the number of

449 clinical strains that were available for comparative analysis was low $(n=22)$, these

450 findings are relevant from a public health perspective. Tetracyclines are highly important

451 antimicrobials [19] since they are among the few alternatives to treat human infections

452 caused by Brucella spp., a pathogen that is associated with cattle. 
453 The rate of resistance to penicillins observed here was not as high as that reported

454 recently in NTS isolated from beef in Mexico [14, 15, 17, 43]. However, this variation is

455 likely associated with the region where isolates originated. Hence, the relevance of

456 cattle as a relevant source of penicillin resistant NTS could not be discarded, as shown

457 by the genomic comparison with other public isolates from Mexico conducted here.

458 In beta-lactam resistance, class-C and class-B ESBLs are the leading concerns. Class-

459 C ESBLs confer resistance to most beta-lactams (except carbapenems) and are not

460 inhibited by clavulanic acid, while class-B metallo-beta-lactamases confer the strongest

461 resistance phenotype, involving all known beta-lactams and clavulanic acid [35].

462 Fortunately, resistance to 3GC, 4GC and carbapenems was rare among experimental

463 isolates, which is in line with recent studies [9]. Apparently, food isolates in Mexico, as

464 well as in the US and Europe [40], do not seem to be a significant source of NTS

465 resistant to 3GC/4GC and carbapenems. Still, we observed some strains (10-15\%)

466 showed non-susceptibility to 3GC, 4GC and carbapenems, despite lacking ESBL-

467 encoding genes. This could be the result of selective pressure and may lead to the

468 emergence of resistance against these critically important antimicrobials, as shown by

469 the moderate proportion of isolates (22\%) exceeding the EUCAST's ECOFF value for

470 meropenem. In Mexico, 3GC used in human medicine (i. e. cefotaxime, ceftriaxone) are

471 also approved, besides ceftiofur, to treat several bovine diseases [44]. Ceftiofur is

472 associated with the emergence of ceftriaxone resistance among livestock and poultry

473 NTS in the United States [40]. Hence, it is important to revise approval of both ceftiofur

474 and other 3GC for veterinary use in Mexico. It has been reported that restrictions

475 (voluntary or law-enforced) in 3GC use in the United States and Canada have helped

476 reduce $3 \mathrm{GC}$ resistance among NTS associated with animals [40]. In any case, given the 
477 limited scientific evidence available in Mexico, continuous surveillance of NTS from

478 foods is of upmost importance to support future risk management decisions.

479 We also observed decreased susceptibility to ciprofloxacin among experimental

480 isolates, a phenomenon that has been associated with the presence of PMQR genes.

481 Particularly, the qnrB19 allele, which was present in over $50 \%$ of our experimental

482 isolates, has also been reported as predominant among NTS strains from the United

483 States [40]. Recent studies from Mexico have also documented high rates (36-44\%) of

484 decreased susceptibility to ciprofloxacin in beef NTS isolates [12], although these

485 authors did not determine AMR genotypes. Moreover, a previous study by our research

486 group documented qnr and oqx alleles were widely distributed among NTS isolated

487 from cattle feces, carcasses and ground beef [17]. Furthermore, the genomic AMR

488 profiling of public NTS isolates from Mexico conducted here, showed qnr alleles are

489 widely disseminated among bovine isolates but not in those from vegetables, seafood,

490 aquatic environments or even clinical strains.

491 In the context of Mexico's cattle production, acquisition and conservation of PMQR

492 genes in NTS is likely the result of selective pressure associated with the use of

493 quinolones (i. e. enrofloxacin), which are approved to treat bovine respiratory, digestive,

494 urinary and skin diseases, among others [44]. Since PMQR genes are carried in

495 plasmids, this increases the risk of their dissemination under positive selective pressure.

496 Many studies report quinolone resistance is rare in NTS isolated from cattle [3, 45].

497 Hence, it has been suggested that the use of these drugs in cattle could not be linked to 498 the emergence of quinolone resistance among cattle NTS isolates [1]. However, PMQR

499 genes are known to confer low-level quinolone resistance, which usually goes

500 undetected when using CLSI breakpoints [46]. Therefore, we believe the role of PMQR 
501 genes as a contributing factor to quinolone resistance in NTS from cattle should not be

502 minimized. Especially, considering PMQR genes have been associated with increasing

503 resistance to quinolones in NTS isolated from foods [47, 48]. In summary, cattle appear

504 to be a relevant source of low-level quinolone resistant NTS. However, to which extent

505 this could result in the emergence of clinical fluoroquinolone resistance among cattle

506 NTS isolates is yet to be determined.

507 Regarding aminoglycoside resistance, although the number of non-susceptible isolates

508 in the experiment was low, the CLSI guidelines emphasize these antimicrobials may

509 appear active in vitro but are not effective clinically against Salmonella and, thus,

510 susceptible isolates should not be reported as such [21]. This may explain why isolates

511 carrying aadA and aph alleles exhibited susceptible phenotypes, a phenomenon that

512 has been observed in previous experiments [17]. Hence, it appears that WGS is a better

513 tool to monitor aminoglycoside resistance as compared to AST. In that sense, our

514 comparative genomics of public NTS from Mexico showed aminoglycoside resistant is

515 less frequent in isolates from food related sources as compared to clinical cases.

516 Resistance to chloramphenicol and trimethoprim-sulfamethoxazole was also frequently

517 observed among experimental isolates (approximately 17 and $20 \%$, respectively).

518 These phenotypes were sustained by acquired AMR genes (i. e. cat, flo, sul, and dfrA

519 alleles). In the case of chloramphenicol, ramR mutations, which are known to confer

520 phenicol resistance [36], seemed to play a role as well.

521 These findings could be explained by the selective pressure exerted by the use of these

522 drugs in food-producing animals. For instance, trimethoprim-sulfamethoxazole is

523 approved as a wide spectrum antibiotic to treat all sorts of bacterial infections in

524 livestock and poultry in Mexico [44]. Likewise, although chloramphenicol is no longer 
525 approved for these purposes, there are other phenicols (i. e. florfenicol) registered. This

526 is likely the reason why phenicol resistance is consistently reported in NTS from animal

527 foods in Mexico, in proportions that vary from moderate $(16-23 \%)[12,15,39]$ to very

528 high $(>90 \%)[14,43]$. Our comparative genomics further supported this analysis since

529 floR, which confer resistance to both phenicols [49], was the dominant resistance factor

530 harbored by NTS from food related sources and clinical cases in Mexico.

531 Regarding MDR profiles, it is interesting to note the higher frequency of MDR isolates in

532 ground beef as compared to lymph nodes. Perhaps this was influenced by the high

533 number of MDR serovar Typhimurium isolates that were exclusively present in ground

534 beef. However, some other factors could further explain these findings. For instance,

535 research on ground beef consistently report high rates of MDR profiles in NTS,

536 regardless of the relative serovar representation $[12,13,15]$. Conversely, studies

537 involving NTS isolated from bovine lymph nodes usually report low levels of MDR

538 phenotypes $[8,50]$. To date, researchers have not been able to decipher the route of

539 entry of NTS circulating in bovine lymph nodes. It has been suggested that fly bites

540 could directly introduce the pathogen into the host's blood [51]. Since this is reasonably

541 likely to occur in feedlots, and NTS survives within the host cells [52], which are not

542 affected by antibiotic treatments, these isolates may not be exposed to the same

543 antimicrobial selective pressure as those circulating in the feces. Hence, they exhibit a

544 weaker AMR profile. This study confirmed pan-susceptible phenotypes are more

545 common among NTS isolates from bovine lymph nodes, while those from ground beef

546 tend to have stronger AMR profiles, as well as higher rates of MDR phenotypes.

547 Among the genetic determinants associated with MDR phenotypes, SGI1 was the most

548 frequently observed. It was present in all MDR serovar Typhimurium isolates (8/10), 
549 which accounted for $40 \%$ of the total number of MDR strains. The SGI 1 contains a 550 class-1 integron and multiple AMR gene cassettes (aadA2, floR, tetG, bla-CARB-2,

551 sul1) conferring resistance to ampicillin, chloramphenicol, streptomycin, sulfonamide,

552 and tetracycline [53]. This penta-resistance profile is typical of MDR S. enterica ser.

553 Typhimurium DT104 carrying SGI1 [29] and was very similar to that observed here.

554 Although SGI1 is not self-mobilizable, it can be transferred to other hosts by donor cells

555 harboring conjugative plasmids [54]. In fact, SGI1 has been recognized as a key factor

556 for the rapid dissemination of strains [55]. Unfortunately, although serovar Typhimurium

557 is known to be dominant in NTS circulating in foods and clinical cases in Mexico, it has

558 been poorly characterized in terms of its genomic AMR profile [9]. According to this

559 review, the SGI1 associated ACSSuT phenotype has been reported in a modest

560 proportion of MDR isolates (9.5\%) collected from clinical cases, chicken, pork, and beef

561 between 2006 and 2013. Analysis of public serovar Typhimurium isolates from Mexico

562 deposited at NCBI $(n=38)$ showed nearly $37 \%$ of them carry AMR genes supporting

563 DT104-like or even stronger phenotypes. Nonetheless, further research is needed to

564 establish if the high rate of DT104-like isolates observed here is a local dissemination

565 phenomenon. Especially, considering half of our MDR Typhimurium isolates also

566 showed decreased susceptibility to ciprofloxacin, which further amplifies the risks pose

567 by these strains to human health. Moreover, it is important to better assess the

568 contribution of point mutations and plasmids to AMR in NTS from foods, which

569 appeared to be modest in the studied sample.

570 In summary, this research showed beef is a moderate source of MDR NTS harboring

571 several AMR genes of human clinical significance. Among the sequenced public NTS

572 isolates from foods and clinical cases in Mexico to date, those from cattle have the 
573 highest proportion of MDR genotypes. However, this should be interpreted with caution

574 since the number of sequenced isolates from other species (i. e. chicken, pigs) and

575 clinical cases is very limited in public databases.

576 Taken together, our results show it is vital to improve NTS control in apparently healthy

577 animals to prevent its dissemination along the food chain and, consequently, human

578 exposure to MDR strains. Particularly, we believe attaining significant improvements in

579 AMR meat safety may require the identification and removal (or treatment) of product

580 harboring MDR NTS, instead of screening for isolates showing resistance to individual

581 antimicrobial classes. Such measures do not seem realistic, in the context of the meat

582 industry of many countries, where testing practices are limited to Salmonella spp.

583 isolation and confirmation. However, most nations have embraced the WHO global

584 action plan on AMR. Thus, they should eventually set up existing technologies, such as

585 WGS, which provide the shortest path to accomplish these goals.

587 Acknowledgments

588 The authors appreciate the technical assistance and training on whole genome

589 sequencing provided by the staff of the National Reference Center for Pesticides and

590 Contaminants belonging to the General Directorate of Agri-Food, Aquaculture and

591 Fisheries Safety of the national Service for Agri-food Health, Safety and Quality. We are

592 also grateful for the support of laboratory technicians, as well as graduate and social

593 service students from the Faculty of Veterinary Medicine, National Autonomous

594 University of Mexico, in field sampling and laboratory analyses. 


\section{$596 \quad$ References}

597 1. Schmidt JW. SMART Antimicrobial Resistance Goals to Drive Meat Safety

598 Improvement. Meat and Muscle Biology. 2020;4(2). doi: 10.22175/mmb.11218.

599 2. Wall BA, Mateus A, Marshall L, Pfeiffer DU, Lubroth J, Ormel HJ, et al. Drivers,

600 dynamics and epidemiology of antimicrobial resistance in animal production.

601 Rome, Italy: Food and Agriculture Organization of the United Nations; 2016.

$602 \quad$ Available from: www.fao.org/publications

603 3. Vikram A, Rovira P, Agga GE, Arthur TM, Bosilevac JM, Wheeler TL, et al. Impact 604 of "Raised without Antibiotics" Beef Cattle Production Practices on Occurrences of 605 Antimicrobial Resistance. Appl Environ Microbiol. 2017;83(22). doi: 10.1128/AEM.01682-17. PMID: 28887421

607 4. Levent G, Schlochtermeier SE, Ives KN, Norman SD, Lawhon GH, Loneragan GH, 608 et al. Population dynamics of Salmonella enterica within beef cattle cohorts 609 followed from single-dose metaphylactic antibiotic treatment until slaughter. Appl $610 \quad$ Environ Microbiol. 2019;85(23): e01386-19. doi:

611 https://doi.org/10.1128/AEM.01386-19. PMID: 31519659

612 5. Ohta N, Norby B, Loneragan GH, Vinasco J, den Bakker HC, Lawhon SD, et al. 613 Quantitative dynamics of Salmonella and E. coli in feces of feedlot cattle treated 614 with ceftiofur and chlortetracycline. PloS One. 2019;14(12): e0225697. doi:

615 10.1371/journal.pone.0225697. PMID: 31791047

616 6. Rovira P, McAllister T, Lakin SM, Cook SR, Doster E, Noyes NR, et al. 617 Characterization of the Microbial Resistome in Conventional and "Raised Without 
618 Antibiotics" Beef and Dairy Production Systems. Front Microbiol. 2019;10: 1980.

619 doi: 10.3389/fmicb.2019.01980. PMID: 31555225

620 7. SSA. Anuarios de Morbilidad 1984-2019: Secretaría de Salud, México; 2019.

$621 \quad$ Available from: https://www.gob.mx/salud/acciones-y-programas/anuarios-de-

622 morbilidad-1984-2019

623 8. Gragg SE, Loneragan GH, Nightingale KK, Brichta-Harhay DM, Ruiz H, Elder JR, 624 et al. Substantial within-animal diversity of Salmonella isolates from lymph nodes, 625 feces, and hides of cattle at slaughter. Appl Environ Microbiol. 2013;79(15): 474450. doi: 10.1128/AEM.01020-13. PMID: 23793628

627 9. Godinez-Oviedo A, Tamplin ML, Bowman JP, Hernandez-Iturriaga M. Salmonella enterica in Mexico 2000-2017: Epidemiology, Antimicrobial Resistance, and Prevalence in Food. Foodborne Pathog Dis. 2020;17(2): 98-118. doi: 10.1089/fpd.2019.2627. PMID: 31647328

10. Laufer AS, Grass J, Holt K, Whichard JM, Griffin PM, Gould LH. Outbreaks of Salmonella infections attributed to beef -United States, 1973-2011. Epidemiol Infect. 2015;143(9): 2003-13. doi: 10.1017/S0950268814003112. PMID: 25427978

11. CDC. Outbreak of Salmonella Infections Linked to Ground Beef: Centers for Disease Control and Prevention (CDC) 2018. Available from: https://www.cdc.gov/salmonella/newport-10-18/index.html

637 12. Realpe-Quintero M, Barba-Leon J, Perez-Montano JA, Pacheco-Gallardo C, 638 Gonzalez-Aguilar D, Dominguez-Arias RM, et al. Genetic diversity and 639 antimicrobial resistance of Salmonella serotypes recovered throughout the beef $640 \quad$ production chain and from patients with salmonellosis. PeerJ. 2018;6: e5482. doi: 
642 13. Cabrera-Diaz E, Barbosa-Cardenas CM, Perez-Montano JA, Gonzalez-Aguilar D,

643 Pacheco-Gallardo C, Barba J. Occurrence, serotype diversity, and antimicrobial resistance of salmonella in ground beef at retail stores in Jalisco state, Mexico. $\mathrm{J}$ Food Prot. 2013;76(12): 2004-10. doi: 10.4315/0362-028X.JFP-13-109. PMID: 24290673

14. Aguilar-Montes de Oca S, Talavera-Rojas M, Soriano-Vargas E, Barba-Leon J, Vazquez-Navarrete J, Acosta-Dibarrat J, et al. Phenotypic and genotypic profile of clinical and animal multidrug-resistant Salmonella enterica isolates from Mexico. J Appl Microbiol. 2018;124(1): 67-74. doi: 10.1111/jam.13615. PMID: 29044980

15. Ballesteros-Nova N, Rubio-Lozano MS, Delgado-Suárez EJ, Méndez-Medina RD, Braña-Varela D, Rodas Suárez O. Perfil de resistencia a antibióticos de serotipos Salmonella spp. aislados de carne de res molida en la Ciudad de México. Salud

16. Quesada A, Reginatto GA, Ruiz Español A, Colantonio LD, Burrone MS. animal para consumo humano. Rev Peru Med Exp Salud Publica. 2016;33(1): 32. doi: 10.17843/rpmesp.2016.331.1899.

17. Delgado Suárez EJ, Ortíz López R, Gebreyes WA, Allard MW, Barona-Gomez F, Salud Rubio MS. Genomic surveillance links livestock production with the emergence and spread of multi-drug resistant non-typhoidal Salmonella in Mexico. J Microbiol. 2019;57(4). doi: DOI 10.1007/s12275-019-8421-3. PMID: 30721457 
666

as public health importance reservoirs of Salmonella spp. Rev Mex Cienc Pecu. 2020;11(3):795-810. doi: 10.22319/rmcp.v11i3.5516.

19. WHO. Critically important antimicrobials for human medicine. 6th Revision 2018: World Health Organization; 2019. Available from: https://www.who.int/foodsafety/publications/antimicrobials-sixth/en/

20. Bauer AW, Kirby WM, Sherris JC, Turck M. Antibiotic susceptibility testing by a standardized single disk method. Am J Clin Pathol. 1966;45(4): 493-6. PMID: 5325707

21. CLSI. Performance Standards for Antimicrobial Susceptibility Testing. 30th ed. CLSI supplement M100. Wayne, PA: Clinical and Laboratory Standards Institute; 2020. Available from: http://em100.edaptivedocs.net/dashboard.aspx

22. EUCAST. MIC and zone diameter distributions and ECOFFs: European Committee on Antimicrobial Susceptibility Testing; 2020. Available from: https://www.eucast.org/mic_distributions_and_ecoffs

23. Magiorakos AP, Srinivasan A, Carey RB, Carmeli Y, Falagas ME, Giske CG, et al. Multidrug-resistant, extensively drug-resistant and pandrug-resistant bacteria: an international expert proposal for interim standard definitions for acquired resistance. Clin Microbiol Infect. 2012;18(3): 268-81. doi: 10.1111/j.14690691.2011.03570.x. PMID: 21793988

24. Andrews S. FastQC: a quality control tool for high throughput sequence data; 2010. Available from: http://www.bioinformatics.babraham.ac.uk/projects/fastqc 25. Bolger AM, Lohse M, Usadel B. Trimmomatic: A flexible trimmer for Illumina Sequence Data. Bioinformatics. 2014;btu170. doi: 10.1093/bioinformatics/btu170. PMID: 24695404 
691 26. Wattam AR, Abraham D, Dalay O, Disz TL, Driscoll T, Gabbard JL, et al. PATRIC, the bacterial bioinformatics database and analysis resource. Nucleic Acids Res. 2014;42(D1): D581-D91. doi: 10.1093/nar/gkt1099. PMID: 24695404

27. Bankevich A, Nurk S, Antipov D, Gurevich AA, Dvorkin M, Kulikov AS, et al. SPAdes: a new genome assembly algorithm and its applications to single-cell sequencing. J Comput Biol. 2012;19(5): 455-77. doi: 10.1089/cmb.2012.0021. PMID: 22506599

28. Feldgarden M, Brover V, Haft DH, Prasad AB, Slotta DJ, Tolstoy I, et al. Validating the AMRFinder Tool and Resistance Gene Database by Using Antimicrobial Resistance Genotype-Phenotype Correlations in a Collection of Isolates.

29. Leekitcharoenphon P, Hendriksen RS, Le Hello S, Weill FX, Baggesen DL, Jun Antimicrob Agents Chemother. 2019;63(11). doi: 10.1128/AAC.00483-19. PMID: SR, et al. Global Genomic Epidemiology of Salmonella enterica Serovar doi: 10.1093/nar/gku985. PMID: 25336619 
714 32. Carattoli A, Zankari E, García-Fernández A, Larsen MV, Lund O, Villa L, et al. In

715 Silico Detection and Typing of Plasmids using PlasmidFinder and Plasmid

716 Multilocus Sequence Typing. Antimicrob Agents Chemother. 2014;58(7): 3895-

717 903. doi: 10.1128/AAC.02412-14. PMID: 25336619

718 33. Babicki S, Arndt D, Marcu A, Liang Y, Grant JR, Maciejewski A, et al. Heatmapper: web-enabled heat mapping for all. Nucleic Acids Res. 2016;44(W1): W147-53. doi:

34. Koutsolioutsou A, Martins E, White DG, Levy SB, Demple B. A soxRS-Constitutive Mutation Contributing to Antibiotic Resistance in a Clinical Isolate of Salmonella enterica (Serovar Typhimurium). Antimicrob Agents Chemother. 2001;45(1): 3843. doi: 10.1128/AAC.45.1.38-43.2001. PMID: 11120941

35. Lee JH, Takahashi M, Jeon JH, Kang LW, Seki M, Park KS, et al. Dual activity of PNGM-1 pinpoints the evolutionary origin of subclass B3 metallo-beta-lactamases: a molecular and evolutionary study. Emerg Microbes Infect. 2019;8(1): 1688-700. doi: 10.1080/22221751.2019.1692638. PMID: 31749408

36. Abouzeed YM, Baucheron S, Cloeckaert A. ramR mutations involved in effluxmediated multidrug resistance in Salmonella enterica serovar Typhimurium.

733 37. Zaidi MB, McDermott P, Fedorka-Cray P, Leon V, Canche C, Hubert S, et al. $734 \quad$ Nontyphoidal Salmonella from human clinical cases, asymptomatic children and 735 raw retail meats in Yucatan, Mexico. Clin Infect Dis. 2006;42: 21-8. doi: 
737 38. Miranda JM, Mondragón AC, Martínez B, Guarddon M, Rodríguez JA. Prevalence

39. Perez-Montaño JA, González-Aguilar D, Barba J, Pacheco-Gallardo C, Campos028X-72.5.966. PMID: 19517722

$$
\text { Bravo CA, García S, et al. Frequency and Antimicrobial Resistance of Salmonella }
$$
Serotypes on Beef Carcasses at Small Abattoirs in Jalisco State, Mexico. J Food Prot. 2012;75(5): 867-73. doi: 10.4315/0362-028X.JFP-11-423. PMID: 22564935

40. McDermott PF, Zhao S, Tate H. Antimicrobial Resistance in Nontyphoidal Salmonella. Microbiol Spectr. 2018;6(4). doi: 10.1128/microbiolspec.ARBA-00142017. PMID: 30027887

41. Nguyen F, Starosta AL, Arenz S, Sohmen D, Donhofer A, Wilson DN. Tetracycline antibiotics and resistance mechanisms. Biol Chem. 2014;395(5): 559-75. doi: 10.1515/hsz-2013-0292. PMID: 24497223

42. Liu YY, Chen CC. Computational Analysis of the Molecular Mechanism of RamR Mutations Contributing to Antimicrobial Resistance in Salmonella enterica. Sci Rep. 2017;7(1): 13418. doi: 10.1038/s41598-017-14008-5. PMID: 29042652

754 43. Villalpando-Guzman S, Vázquez-Quiñones CR, Natividad-Bonifacio I, Curiel755 Quesada E, Quiñones-Ramírez El, Vázquez-Salinas C. Frecuencia, 756 susceptibilidad antimicrobiana y patrón de adherencia de Salmonella enterica 757 aislada de carne de pollo, res y cerdo de la Ciudad de México. Rev Chilena 758 Infectol. 2017;34(5): 458-66. doi: 10.4067/S0716-10182017000500458. PMID: 
760 44. SADER. Productos químico-farmacéuticos vigentes: Secretaría de Agricultura y

761 Desarrollo Rural, Gobierno de México; 2020. Available from:

762

763 https://www.gob.mx/cms/uploads/attachment/file/512374/PRODUCTOS_VIGENT

45. Smith AB, Renter DG, Cernicchiaro N, Shi X, Nagaraja TG. Prevalence and Quinolone Susceptibilities of Salmonella Isolated from the Feces of Preharvest Cattle Within Feedlots that Used a Fluoroquinolone to Treat Bovine Respiratory Disease. Foodborne Pathog Dis. 2016;13(6): 303-8. doi: 10.1089/fpd.2015.2081.

46. Strahilevitz J, Jacoby GA, Hooper DC, Robicsek A. Plasmid-mediated quinolone resistance: a multifaceted threat. Clin Microbiol Rev. 2009;22(4): 664-89. doi:

47. Pribul BR, Festivo ML, Rodrigues MS, Costa RG, Rodrigues EC, de Souza MM, et al. Characteristics of Quinolone Resistance in Salmonella spp. Isolates from the Food Chain in Brazil. Front Microbiol. 2017;8: 299. doi: 10.3389/fmicb.2017.00299.

$$
\text { PMID: } 28352250
$$

48. Karp BE, Campbell D, Chen JC, Folster JP, Friedman CR. Plasmid-mediated quinolone resistance in human non-typhoidal Salmonella infections: An emerging public health problem in the United States. Zoonoses Public Health. 2018;65(7): 838-49. doi: 10.1111/zph.12507. PMID: 30027554

49. Arcangioli MA, Leroy-Sétrin S, Martel JL, Chaslus-Dancla E. A new chloramphenicol and £orfenicol resistance gene flanked by two integron structures in Salmonella typhimurium DT104. FEMS Microbiol Lett. 1999;74: 327-32. doi: 
784 50. Gragg SE, Loneragan GH, Brashears MM, Arthur TM, Bosilevac JM,

$785 \quad$ Kalchayanand N, et al. Cross-sectional study examining Salmonella enterica

786 carriage in subiliac lymph nodes of cull and feedlot cattle at harvest. Foodborne

787 Pathog Dis. 2013;10(4): 368-74. doi: 10.1089/fpd.2012.1275. PMID: 23566273

788 51. Olafson PU, Brown TR, Lohmeyer KH, Harvey RB, Nisbet DJ, Loneragan GH, et

789 al. Assessing Transmission of Salmonella to Bovine Peripheral Lymph Nodes

790 upon Horn Fly Feeding. J Food Prot. 2016;79(7): 1135-42. doi: 10.4315/0362-

791 028X.JFP-15-414. PMID: 27357032

792 52. OhI ME, Miller SI. SALMONELLA: A Model for Bacterial Pathogenesis. Annu Rev

793 Med. 2001;52: 259-74. doi: 10.1146/annurev.med.52.1.259. PMID: 11160778

794 53. Mulvey MR, Boyd DA, Olson AB, Doublet B, Cloeckaert A. The genetics of

795 Salmonella genomic island 1. Microbes Infect. 2006;8(7): 1915-22. doi:

796 10.1016/j.micinf.2005.12.028. PMID: 16713724

797 54. Hall R. Salmonella genomic islands and antibiotic resistance in Salmonella

798 enterica. Future Microbiol. 2010;5(10): 1525-38. doi: 10.2217/fmb.10.122. PMID:

21073312

800 55. Levings RS, Lightfoot D, Partridge SR, Hall RM, Djordjevic SP. The genomic

801 island SGI1, containing the multiple antibiotic resistance region of Salmonella

802 enterica serovar Typhimurium DT104 or variants of it, is widely distributed in other

803 S. enterica serovars. J Bacteriol. 2005;187(13): 4401-9. doi:

$804 \quad$ 10.1128/JB.187.13.4401-4409.2005. PMID: 15968049 


\section{Supporting information}

806 S1 Table. NCBI accessions and metadata of our fully sequenced Salmonella isolates

807 collected from bovine lymph nodes and ground beef (XLSX).

808

809 S2 Table. NCBI accessions, metadata and antimicrobial resistance genotypes of fully sequenced public Salmonella isolates from Mexico included in this study

812 S3 Table. NCBI accesions, metada and antimicrobial resistance genotypes of fully

813 sequenced public Salmonella enterica ser. Typhimurium isolates from Mexico included

814 in this study

816 S1 Figure. BLAST atlas of Salmonella virulence plasmid pSLT of ten experimental

817 serovar Typhimurium isolates. The black slot corresponds to the backbone and the

818 inner ring to the reference plasmid sequence and genes. Map generated with GView,

819 version 1.7 through the tblastx program, with e-value $=0.001$, alignment cutoff $=50$,

820 identity cutoff $=70$, and no filtering of low complexity sequences. Refer to $\mathrm{S} 1$ Table for

821 isolates accessions and metadata.

823 S2 Figure. BLAST atlas of plasmid pK245 of one experimental serovar Give isolate. The

824 black slot corresponds to the backbone and the inner ring to the reference plasmid

825 sequence and genes. Map generated with GView, version 1.7 through the tblastx

826 program, with e-value $=0.001$, alignment cutoff $=50$, identity cutoff $=70$, and no filtering of

827 low complexity sequences. Refer to Table S1 for isolate accession and metadata. 
829 S3 Figure. BLAST atlas of plasmid R64 of experimental serovar Fresno and Anatum

830 isolates. The black slot corresponds to the backbone and the inner ring to the reference

831 plasmid sequence and genes. Map generated with GView, version 1.7 through the

832 tblastx program, with e-value $=0.001$, alignment cutoff $=50$, identity cutoff $=70$, and no

833 filtering of low complexity sequences. Refer to S1 Table for isolate accessions and

834 metadata.

836 S4 Figure. BLAST atlas of plasmid pOLA52 of one experimental serovar monophasic

837 Typhimurium isolate. The black slot corresponds to the backbone and the inner ring to

838 the reference plasmidsequence and genes. Map generated with GView, version 1.7

839 through the tblastx program, with e-value=0.001, alignment cutoff $=50$, identity cutoff $=70$,

840 and no filtering of low complexity sequences. Refer to Table S1 for isolate accession and 841 metadata.

\section{Author Contributions}

843 Conceptualization: María Salud Rubio Lozano, Orbelín Soberanis Ramos, Rubén

844 Danilo Méndez Medina, Enrique Jesús Delgado Suárez.

845 Data curation: Tania Palós Gutiérrez, Francisco Alejandro Ruíz López, Cindy Fabiola

846 Hernández Pérez.

847 Formal analysis: Tania Palós Gutiérrez, Cindy Fabiola Hernández Pérez, Nayarit

848 Emérita Ballesteros Nova, Enrique Jesús Delgado Suárez.

849 Funding acquisition: María Salud Rubio Lozano, Orbelín Soberanis Ramos, Marc W.

850 Allard. 
851 Methodology: Cindy Fabiola Hernández Pérez, Francisco Alejandro Ruíz López,

852 Enrique Jesús Delgado Suárez.

853 Project administration: María Salud Rubio Lozano, Orbelín Soberanis Ramos.

854 Resources: María Salud Rubio Lozano, Orbelín Soberanis Ramos, Francisco Alejandro

855 Ruíz López.

856 Software: Cindy Fabiola Hernández Pérez, Nayarit Emérita Ballesteros Nova, Enrique

857 Jesús Delgado Suárez.

858 Supervision: María Salud Rubio Lozano, Rubén Danilo Méndez Medina, Marc W.

859 Allard, Enrique Jesús Delgado Suárez.

860 Visualization: Tania Palós Gutiérrez, Enrique Jesús Delgado Suárez.

861 Writing - original draft: Tania Palós Gutiérrez, Enrique Jesús Delgado Suárez.

862 Writing - review \& editing: Enrique Jesús Delgado-Suárez, Tania Palós-Guitérrez,

863 Francisco Alejandro Ruíz-López, Cindy Fabiola Hernández Pérez, Nayarit Emérita

864 Ballesteros-Nova, Orbelín Soberanis-Ramos, Rubén Danilo Méndez-Medina, Marc W.

865 Allard, María Salud Rubio-Lozano. 


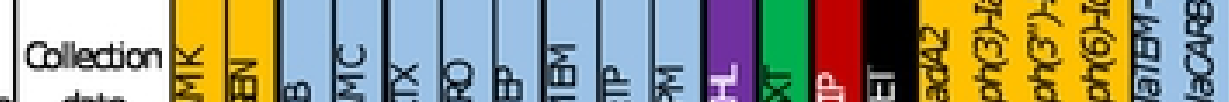

AKG9 Anatum

AKDO Anatum

AKV1 Anatum

AK/2 Anatum

AK/3 Anatum

AK04 Anatum

AKF5 Anatum

AK76 Anatum

Alas Anatum

AKA9 Anatum

\begin{tabular}{lll}
\hline AKB1 Anatum & IN & $5 / 10 / 17$
\end{tabular}

\begin{tabular}{l|ll}
\hline AKBS Anatum & GB & $5 / 22 / 17$ \\
\hline
\end{tabular}

AM39 Anatum 1 UN $5 / 24 / 17$

\begin{tabular}{llll}
\hline AM 40 Anatum & G & $5 / 24 / 17$ \\
\hline
\end{tabular}

AM44 Aratum 1 IN $6 / 5 / 17$

\begin{tabular}{lll}
\hline AM46 Anatum & IN & $6 / 12 / 17$ \\
\hline
\end{tabular}

\begin{tabular}{l|l|l}
\hline AM58 Anatum & LN & $8 / 9 / 17$ \\
\hline
\end{tabular}

\begin{tabular}{l|l|l}
\hline AM68 Anatum & UN & $9 / 25 / 17$
\end{tabular}

\begin{tabular}{lll}
\hline AM79 Anatum & IN & $10 / 16 / 17$ \\
\hline
\end{tabular}

AM89 Anatum 1 IN $4 / 23 / 18$

AM90 Aratum 1 GB $4 / 23 / 18$

\begin{tabular}{l|ll}
\hline AM95 Anatum & IN & $423 / 18$ \\
\hline
\end{tabular}

AM98 Anatum UN 4/30/18

\begin{tabular}{l|l|l}
\hline AKBO Fresno & GB & $5 / 8 / 17$ \\
\hline
\end{tabular}

AM78 Fresno

AM86 Fresno

\begin{tabular}{l|l|l} 
& G & $4 / 9 / 18$
\end{tabular}

\begin{tabular}{|l|c|c|}
\hline AS56 Give & UN & $9 / 24 / 18$ \\
\hline AN13_I4,55,12:i:- & UN & $5 / 7 / 18$ \\
\hline
\end{tabular}

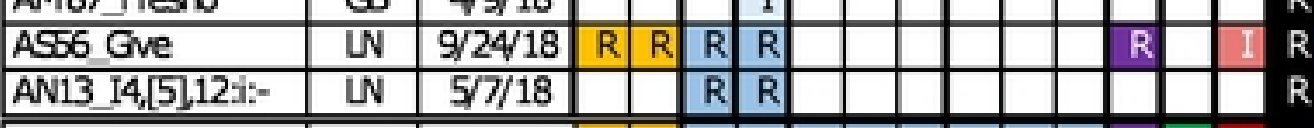

ID serovar

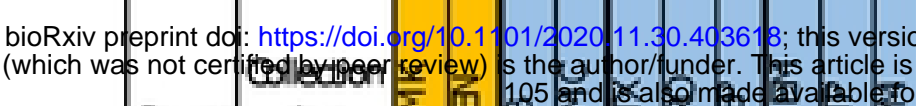

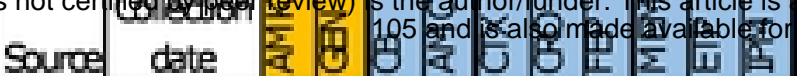

\begin{tabular}{l|l|l}
\hline ANO3 Kentudky & LN & $5 / 7 / 18$ \\
\hline
\end{tabular}

AN22 Kentudy $\quad$ GB $5 / 28 / 18$

\begin{tabular}{lll}
\hline AN23 Kentudy & IN & $5 / 28 / 18$ \\
\hline
\end{tabular}

\begin{tabular}{l|l|l}
\hline AN24 Kentucky & IN & $6 / 4 / 18$ \\
\hline
\end{tabular}

\begin{tabular}{l|l|l|}
\hline AN27 Kentudky & IN & $6 / 4 / 18$ \\
\hline
\end{tabular}

\begin{tabular}{|l|l|l|}
\hline AK57_London & LN & $4 / 3 / 17$ \\
\hline AM45
\end{tabular}

\begin{tabular}{l|l|l}
\hline AM45 London & WN & $6 / 12 / 17$ \\
\hline
\end{tabular}

AM47 London 1 WN $6 / 12 / 17$

\begin{tabular}{l|l|l}
\hline AM49 London & LN & $8 / 2 / 17$ \\
\hline
\end{tabular}

AM50 London $\quad$ LN $8 / 2 / 17$

\begin{tabular}{|l|l|l|}
\hline AM63 London & LN & $9 / 11 / 17$ \\
\hline AM74 London & WN & $10 / 9 / 17$ \\
\hline
\end{tabular}

\begin{tabular}{l|l|l}
\hline ANO4 London & $G$ & $5 / 7 / 18$ \\
\hline
\end{tabular}

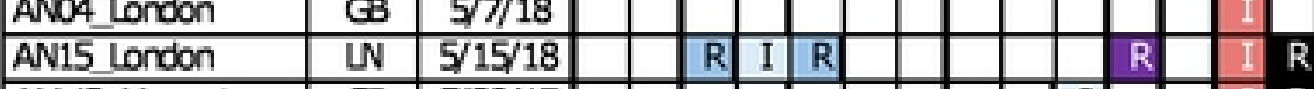

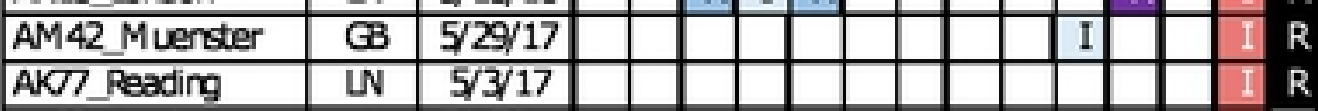

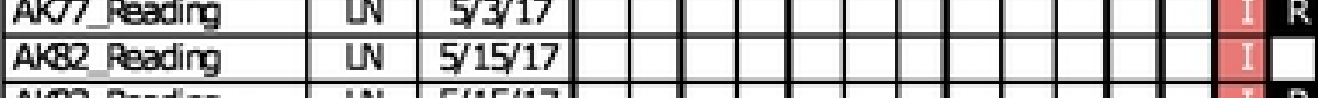

AKB3 Peading $\quad$ IN $5 / 15 / 17$

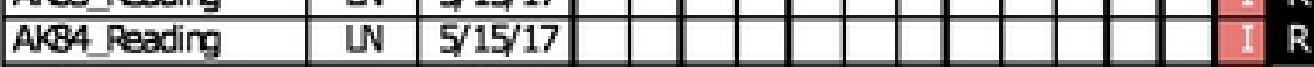

\begin{tabular}{l|l|l}
\hline AKB6 Peading & IN $5 / 22 / 17$ \\
\hline
\end{tabular}

AM36 peading $\quad$ GB $5 / 22 / 17$

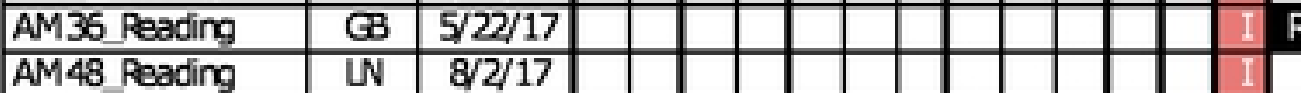

\begin{tabular}{l|c|c}
\hline AM51 Peading & G & $8 / 2 / 17$ \\
\hline
\end{tabular}

\begin{tabular}{l|ll}
\hline AM52 Peading & GB & $8 / 8 / 17$
\end{tabular}

\begin{tabular}{l|l|l}
\hline AM53 Peading & GB & $8 / 8 / 17$ \\
\hline
\end{tabular}

\begin{tabular}{l|c|c}
\hline AM54 peading & G & $8 / 8 / 17$
\end{tabular}

\begin{tabular}{l|l|l}
\hline AM55 Peading & IN & $8 / 8 / 17$ \\
\hline
\end{tabular}

AM56 peading

D serovar

AM57_Reading

AM60 peading

AM83 Peading

ANO5 Reading

AN06 Reading

AN12 Reading

AN14 Reading

AN26 Reading

AN31 Reading

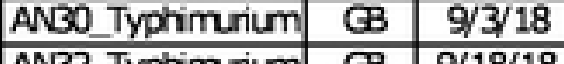

AN32 Typhimurium GB $9 / 18 / 18$

\begin{tabular}{|l|l|l|}
\hline AN33_Typhimurium & G & $9 / 18 / 18$ \\
\hline AN34 Typhimurium & GB & $9 / 18 / 18$ \\
\hline
\end{tabular}

\begin{tabular}{|l|l|l}
\hline AN34_Typhimurium & GB & $9 / 18 / 18$ \\
\hline
\end{tabular}

\begin{tabular}{|l|l|l}
\hline AN35 Typhimurium & GB & $9 / 18 / 18$ \\
\hline
\end{tabular}

\begin{tabular}{|l|l|l|}
\hline AN36 Typhimurium & G & $9 / 18 / 18$ \\
\hline AN
\end{tabular}

\begin{tabular}{ll|l|}
\hline AN37 Typhimurium & GB & $9 / 18 / 18$ \\
\hline
\end{tabular}

AN38 Typhimurium GB $9 / 18 / 18$

\begin{tabular}{|l|l|l|}
\hline AN39_Typhimurium & GB & $9 / 18 / 18$ \\
\hline
\end{tabular}

Fig1

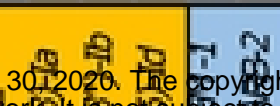

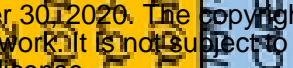
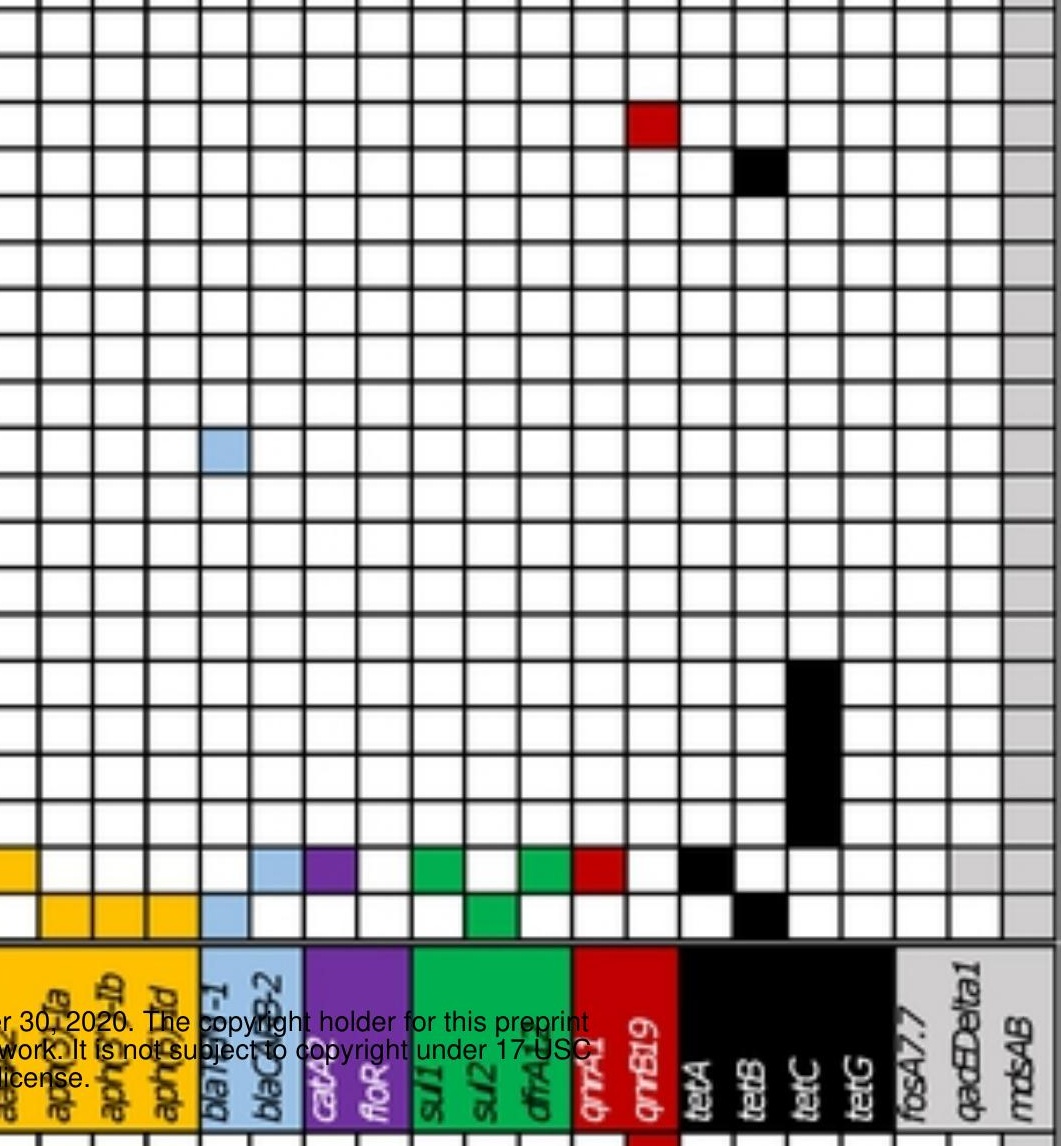

है

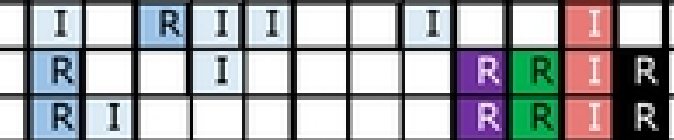

\begin{tabular}{lllllllllllll}
$R$ & $I$ & & & & & & & $R$ & $R$ & $I$ & $R$ \\
\hline
\end{tabular}

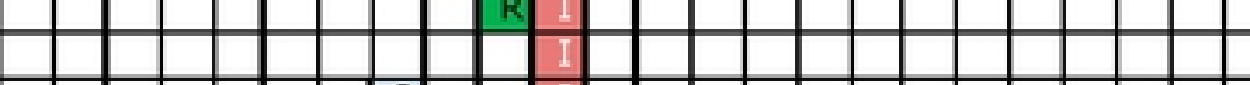

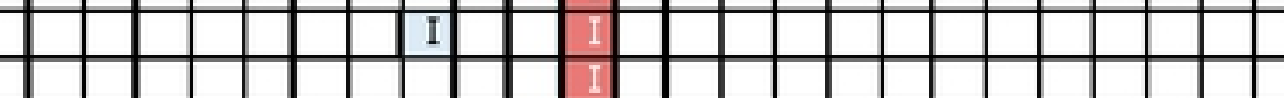

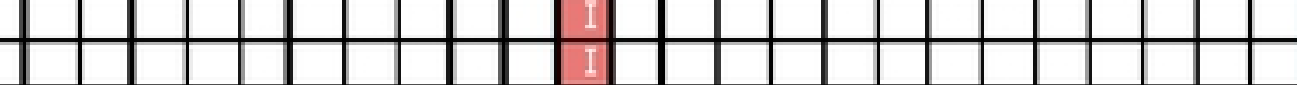

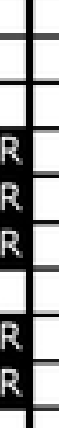

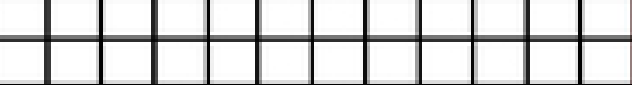

\begin{tabular}{llllllllllll}
\hline & & & & & & & & & & & \\
\hline
\end{tabular}

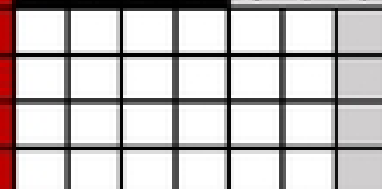

\begin{tabular}{lllllllllll} 
& & I & R & & I & I & & I \\
\hline
\end{tabular}

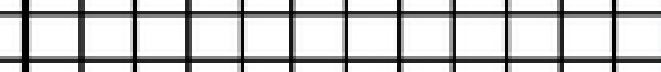

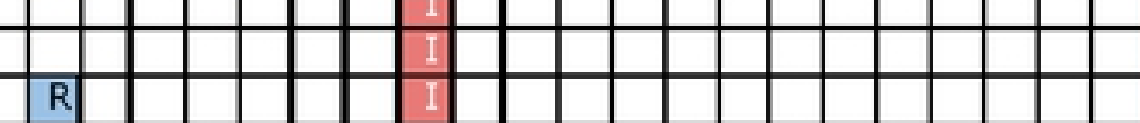

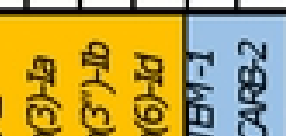
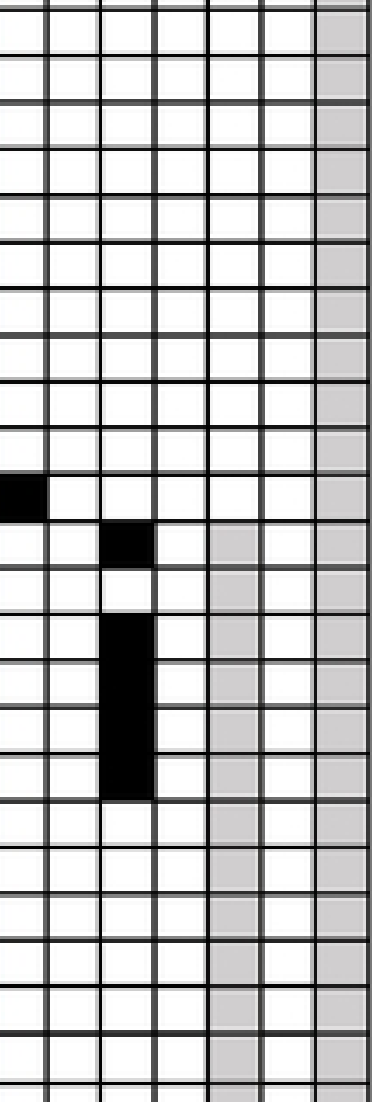

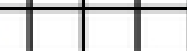

हี 


\section{aMR genotypes $\approx$ AMR phenotypes}

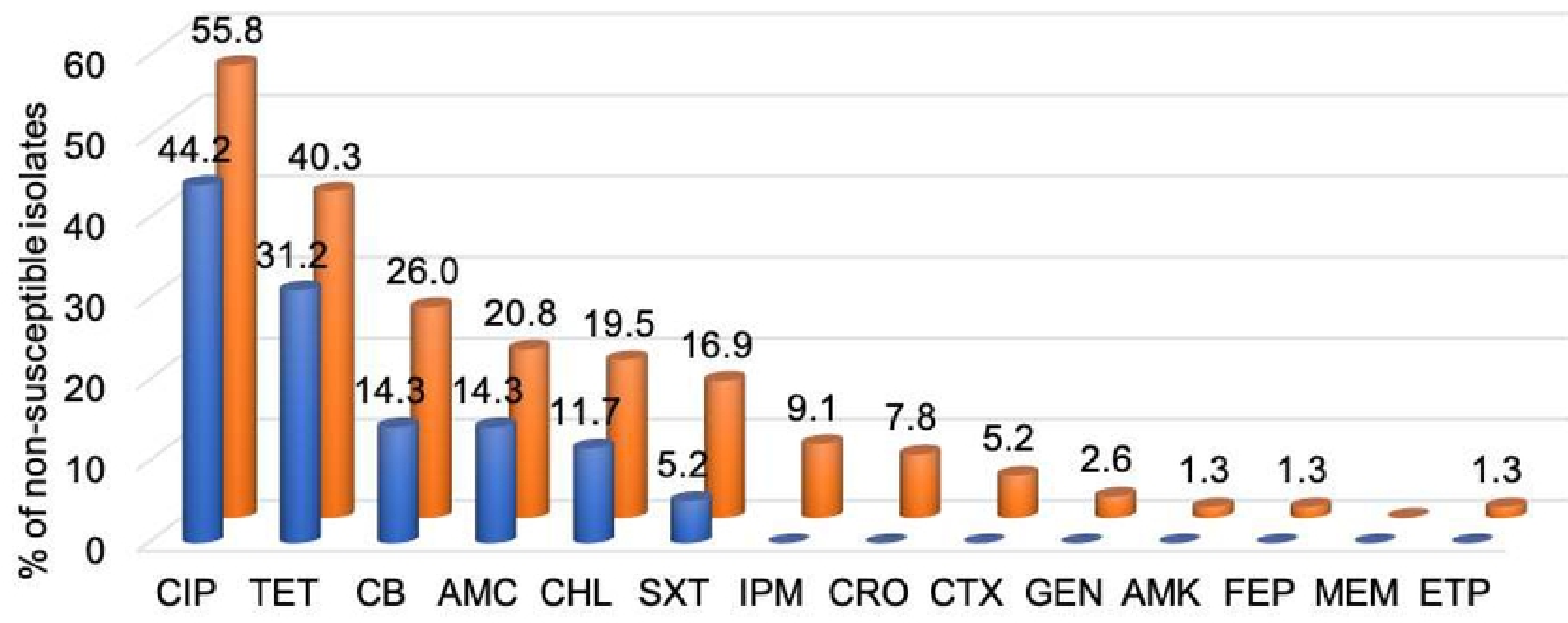

Fig2 


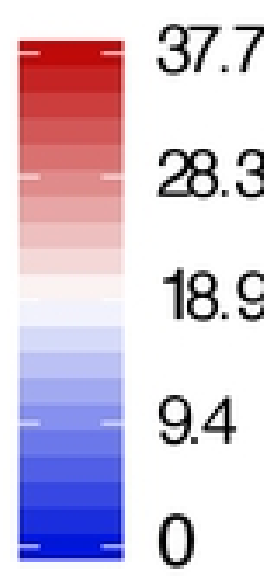

Clinical cases $(n=22)$

Bovines $(n=114)$

Vegetables $(n=1064)$

Seafood $(n=129)$

Aquatic environments $(n=385)$

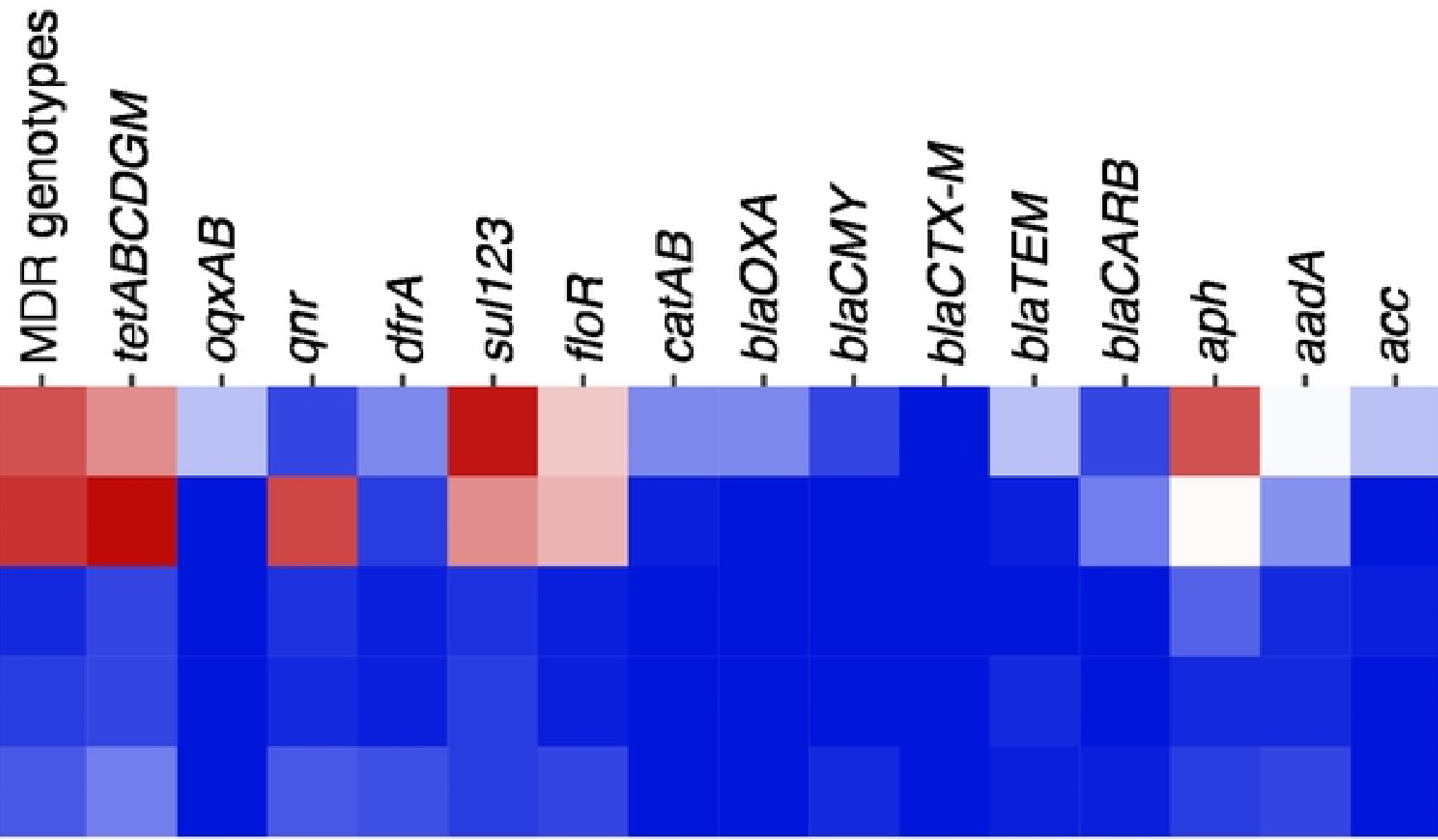

Fig3 
